\title{
ELABORAÇÃO DE UM QUEIJO ANÁLOGO AO PECORINO ROMANO PRODUZIDOO COM LEITE DE CABRA E COALHADA CONGELADOS
}

\author{
Elaboration of a similar cheese to the pecorino romano made with frozen goat milk and frozen curd
}

\author{
Roberto Abdallah Curi' ${ }^{1}$, Ismael Antonio Bonassi ${ }^{2}$
}

\begin{abstract}
RESUMO
A pequena produção por animal e a sazonalidade são fatores limitantes na utilização de leite de cabra, com conseqüente descontinuidade no fornecimento de seus produtos. Isto ocorre mesmo em países que possuem uma caprinocultura leiteira importante, localizados em clima temperado. Em função disso, objetivou-se com o presente trabalho verificar a possibilidade de fabricar um produto similar ao queijo Pecorino Romano, utilizando-se leite de cabra congelado e coalhada congelada caracterizando os queijos resultantes, do ponto de vista organoléptico relacionado com a cura. $\mathrm{O}$ delineamento experimental foi estabelecido para verificar o efeito do congelamento lento e rápido do leite de cabra e da coalhada nas características sensoriais do produto, efetuando-se os seguintes tratamentos: Tratamento 1 - queijos elaborados sem congelamento do leite e da coalhada, (S.C.); Tratamento 2 - queijos elaborados com congelamento lento da coalhada obtida, (C.L.C.); Tratamento 3 - queijos elaborados com congelamento rápido da coalhada obtida, utilizando-se nitrogênio líquido, (C. R. C.); Tratamento 4 - queijos elaborados após o congelamento rápido do leite, utilizando-se nitrogênio líquido (C.R.L.); Tratamento 5 - queijos elaborados após o congelamento lento do leite, (C.L.L.). A análise sensorial foi realizada, depois de um período de dois meses de maturação dos queijos, por um painel composto por 10 provadores selecionados e treinados. Para o grupo de atributos analisados foi possível considerar que o tratamento 4 teve o melhor desempenho com médias mais altas para aroma, sabor e escala hedônica e médias mais baixas para gosto ácido, sabor estranho e olhaduras mecânicas e ou gasosas. Esse tratamento mostrou, também, média baixa para sabor picante, textura e consistência. O pior desempenho foi o do tratamento 3, ainda assim, com resultados médios que não o desqualificaram totalmente. Portanto, o produto estudado poderia utilizar leite de cabra nas condições naturais (T1) nas épocas de pico da produção e utilizar congelamento lento do leite (T5), que seria realizado na época de pico da produção, na entressafra.
\end{abstract}

Termos para indexação: Leite de cabra, queijo, leite de cabra congelado, coalhada congelada.

\section{ABSTRACT}

Seasonal production and small yield per animal are limiting factors when using goat's milk with consequent problems in the products supply, even in countries with well developed milk goat's herds located in temperate zones. The aim of this work was the production of a similar cheese to the "Pecorino Romano" using frozen goat's milk and frozen curd. The resultant cheeses was characterizing of the point of view organoléptic related with the cure. The experimental line was established to verify the effect of slow freezing and quick freezing of goat milk and curd in the sensory characteristics of the product, using the following treatments: Treatment 1 - milk and curd without freezing, (N.F.); Treatment 2 - slow freezing of the obtained curd, (S.F.C.); Treatment 3 - quick freezing of the obtained curd, using liquid nitrogen, (Q.F.C.); Treatment 4 - quick freezing of milk, using liquid nitrogen, (Q.F.M.); Treatment 5 - slow freezing of milk, (S.F.M.). The sensory evaluation was performed after a 2 month period of cheese ripening by a panel composed by 10 tasters selected and trainned. The results indicated that there was a significant statistic difference among the treatments when submitted to a sensory evaluation after cheese ripening. For the analyzed attribute group it was possibly to consider that the treatment 4 had the best performance with high average for tast, flavor and hedonic scale and low average for acid flavor, off flavor, mechanic holes and or gaseous. This treatment showed, also, average low for spiced flavor, texture e consistency. The worst performance was for treatment 3 but the medium results did not disqualify it totally. Therefore, for the production of the studied cheese, goat's milk in natural conditions could be used (T1) during high milk production season and previously slow frozen milk, during small milk production season (T5).

Index terms: Goat's milk, cheese, frozen goat milk, frozen curd.

\section{(Recebido para publicação em 16 de junho de 2004 e aprovado em 5 julho de 2006)}

\section{INTRODUÇÃO}

O queijo é o produto de maior interesse tecnológico e econômico produzido com leite de cabra. $\mathrm{Na}$ França aproximadamente $80 \%$ da produção de leite caprino é transformado em queijo, $45 \%$ dos quais processados na própria fazenda (FURTADO, 1985). Ainda na França, estima-se que, em 1992, 400 milhões de litros de leite caprino foram transformados em queijos (DUMOULIN \& PERETZ, 1993; ZAPICO et al., 1995). Na Espanha 57\% (RAMOS \& JUAREZ, 1993) e na Itália cerca de $63 \%$ do leite é transformado em queijo (GAIFAMI, 1985).

"Aluno do Curso de Pós-Graduação em Agronomia - Área de Concentração em "Energia na Agricultura" - Faculdade de Ciências Agronômicas Campus de Botucatu/UNESP - Rua Miguel Cioffi, 240 - Vila São João - 18.607-693 - Botucatu, SP - curi@fca.unesp.br ${ }^{2}$ Professor Titular Aposentado (Voluntário) do Departamento de Gestão e Tecnologia Agroindustrial - Faculdade de Ciências Agronômicas/UNESP _ Cx. P. 277 - 18603-970 - Botucatu, SP - secdtpa@fca.unesp.br 
Atualmente, grande parte da produção mundial de leite de cabra é destinada à fabricação de queijos. Como a fabricação desses queijos é basicamente artesanal, houve o desenvolvimento de mais de 400 variedades, sendo algumas destas em combinação com leite de vaca, ovelha ou búfala (SINGH et al., 1992).

No Brasil, existe interdição para fabricar queijos com leite cru quando o tempo de maturação for menor que 60 dias a uma temperatura superior a $5^{\circ} \mathrm{C}$ (BRASIL, 2002).

Os diversos tipos de queijo compreendem algumas etapas comuns em sua fabricação. Entre as etapas, ou mesmo durante elas, pode haver variação relativas a tempo de descanso da massa, tempo de mexeduras, diferenças de temperaturas, tempo de dessoragem e, também, diferenças na condição de maturação. Esses fatores determinarão a textura, aroma e sabor de cada queijo, determinando suas diferenças e características.

A pequena produção por animal e a sazonalidade são fatores limitantes, pois não se tem continuidade no fornecimento dos produtos (GOMES et al., 1997). Isto ocorre mesmo em países que possuem uma caprinocultura leiteira importante e estão localizados em regiões de clima temperado. Algumas das formas indicadas para contornar o problema da sazonalidade seriam a regularização do cio das cabras, com a utilização de hormônios e luminosidade artificial (CORDEIRO, 1996); ou a elaboração de queijos de massa cozida “tipo Pecorino Romano". Estes podem ser fabricados na época de pico da produção, entre setembro e novembro, para serem consumidos na entressafra, ou seja, no inverno. Outra alternativa seria o congelamento das massas para serem utilizadas em época oportuna.

Conforme Bonassi et al. (2002), queijos fabricados com coalhada congelada pelo processo lento apresentaram uma textura inferior aos demais tratamentos.

Segundo Yamasaki et al. (2001), os queijos produzidos com coalhada congelada com cura de três meses apresentaram odor rançoso e os queijos de massa congelada com maturação de dois meses tinham o "flavor" similar aos queijos de massa sem congelamento.

Pesquisas têm demonstrado que os efeitos do congelamento - descongelamento, para o leite de cabra, são menos acentuados do que para o leite de vaca. A resolução 93 da Secretaria e Abastecimento do Estado de São Paulo, publicada no Diário Oficial de 14/10/93, regulamentou a comercialização do leite de cabra congelado (SÃO PAULO, 1993).

De acordo com Albuquerque \& Castro (1996), o Pecorino Romano é um queijo de origem italiana sendo que, em princípio, não apresentava tamanho definido, principalmente porque era de fabricação estritamente familiar. Adquiriu fama pela suas características definidas referentes à massa dura e uniforme, aroma atraente e sabor picante.

\section{MATERIAL E MÉTODOS}

Para a elaboração dos queijos foi utilizado leite de cabra da raça Parda Alpina, ordenhadas manualmente, proveniente da Fazenda Experimental Lageado - UNESP.

$\mathrm{O}$ leite foi pasteurizado, à temperatura de $63^{\circ} \mathrm{C}$ durante 30 minutos, no Laboratório de Tecnologia dos Produtos de Origem Animal da Faculdade de Ciências Agronômicas (FCA)-Campus de Botucatu.

Após a pasteurização o leite foi dividido em cinco partes, que constituíram os tratamentos a seguir especificados: Tratamento 1 - queijos elaborados sem congelamento do leite e da coalhada, (S.C.); Tratamento 2 - queijos elaborados com congelamento lento da coalhada obtida, (C.L.C.); Tratamento 3 - queijos elaborados com congelamento rápido da coalhada obtida, utilizando-se nitrogênio líquido, (C.R.C.); Tratamento 4 - queijos elaborados após o congelamento rápido do leite, utilizandose nitrogênio líquido, (C.R.L.); Tratamento 5 - queijos elaborados após o congelamento lento do leite, (C.L.L.). O ensaio foi realizado com queijos distribuídos em quatro blocos com os cinco tratamentos anteriormente especificados.

Foi utilizada cultura lática termofilica (TCC-20), DVS500U (Direct Vat Set) constituída pelas espécies: Streptococcus thermophilus (St), Lactobacillus delbrueckii subespécie bulgaricus (Lb) e Lactobacillus helveticus; cloreto de cálcio, solução a $50 \%$ e, também, coalho líquido produzido por uma cepa de Aspergillus niger var. awamori, poder coagulante 1: 3000.

Para a elaboração dos queijos foi seguida a metodologia indicada em Albuquerque \& Castro (1996) para o queijo tipo Pecorino Romano, conforme o fluxograma a seguir, com as devidas adaptações necessárias ao desenvolvimento proposto, principalmente, tempos de salga e de cura, que foram definidos em função do tamanho dos queijos resultantes.

As análises sensoriais dos queijos foram realizadas no Laboratório de Tecnologia dos Produtos de Origem Animal, conduzidas conforme Institute of Food Technologists (1981) e Meilgaard et al. (1990), em cabinas apropriadas, longe de ruídos e de odores. Foram efetuadas em horários previamente determinados e por provadores selecionados e treinados conforme Roça \& Bonassi (1985). 
As amostras foram servidas à temperatura ambiente, em placas de Petri, codificadas com quatro dígitos e distribuídas ao acaso. Os testes foram realizados utilizandose escala não estruturada de nove centímetros, conforme Meilgaard et al. (1990). Foram anotadas as seguintes variáveis: aroma, variando de zero $(0=$ muito ruim) a nove ( $9=$ muito bom); sabor, variando de zero $(0=$ muito ruim) a nove $(9=$ muito bom); sabor picante variando de zero $(0=$ sem sabor picante) a nove ( $9=$ muito picante); gosto ácido, variando de zero $(0=$ sem gosto ácido $)$ a nove $(9=$ muito ácido); sabor estranho, variando de zero $(0=$ nenhum $)$ a nove ( $9=$ extremamente forte); consistência, variando de zero $(0=$ macia $)$ a nove $(9=$ muito dura $)$; textura, variando de zero $(0=$ não farinácea $)$ a nove $(9=$ farinácea $)$ e presença de olhaduras mecânicas e ou gasosas, variando de zero (0 $=$ ausente $)$ a nove $(9=$ numerosos $)$. Complementando as análises anteriores foi também utilizada escala hedônica, variando de um ( 1 = desgostei extremamente) a nove $(9=$ gostei extremamente). Foram utilizados dez provadores que avaliaram cada um dos 5 tratamentos: queijos elaborados sem congelamento do leite e da coalhada, (T1); queijos elaborados com congelamento lento da coalhada obtida, (T2); queijos elaborados com congelamento rápido da coalhada obtida, utilizando-se nitrogênio líquido, (T3); queijos elaborados com congelamento rápido do leite, utilizando-se nitrogênio líquido, (T4); e queijos elaborados com congelamento lento do leite, (T5).

A avaliação estatística do resultado da análise sensorial foi efetuada utilizando-se Análise de Variância para experimento em blocos aleatorizados, com o cálculo das estatísticas F e p, em que p é a probabilidade de erroneamente concluir pela significância. Nos casos em que $\mathrm{p}<0,05$ foram efetuados contrastes entre médias pelo método de Tukey com o cálculo da diferença mínima significativa para $\alpha=0,05$, conforme Curi (1998).

\section{RESULTADOS E DISCUSSÃO}

Os valores médios obtidos, logo após a maturação dos queijos, para aroma, sabor, sabor picante, gosto ácido, sabor estranho, consistência, textura, presença de olhaduras mecânicas e ou gasosas e para a escala hedônica, estão na Tabela 1. Os resultados da análise estatística, estão na Tabela 2.

\section{Aroma}

Os tratamentos T4, T5 e T1 apresentaram médias maiores que 6,0 com indicação de aroma agradável. O pior desempenho que foi o de T3, com média 5,48, pode ser considerado um resultado de médio a bom indicando que,
Fluxograma para elaboração dos queijos

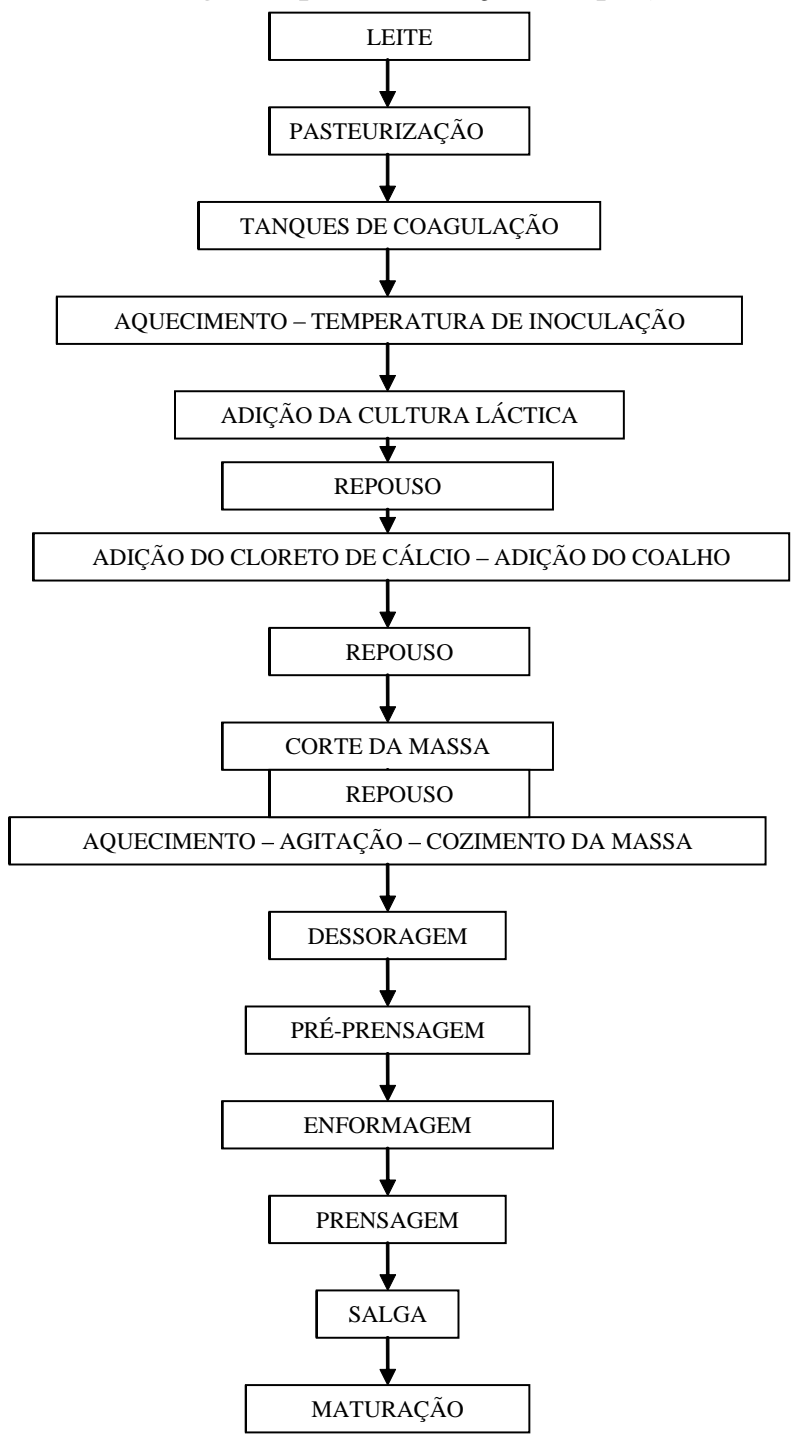

FIGURA 1 - Fluxograma de processamento dos queijos.

de forma geral, todos os tratamentos apresentaram aroma bom o que é interessante, pois o conhecimento popular tende a achar que produtos com leite de cabra apresentam aroma desagradável.

\section{Sabor}

Os melhores sabores ocorreram nos tratamentos T1, T4 e T5, todos com média próxima de 6,0 o que seria indicativo de sabor entre médio e superior. Com o aumento do tempo de maturação do produto o sabor poderia acentuar 
suas características positivas, o que não foi possível verificar neste experimento em que, por razões práticas, o tempo de maturação do produto não pôde ser prolongado.

\section{Sabor picante}

De forma geral, os provadores não identificaram sabor picante acentuado nos queijos dos 5 tratamentos, já

TABELA 1 - Média dos valores das 4 repetições obtidos na avaliação sensorial efetuada logo após a maturação dos queijos para todos os tratamentos.

\begin{tabular}{lccccc}
\hline & \multicolumn{5}{c}{ Tratamentos } \\
\cline { 2 - 6 } Atributo Sensorial & T1 & T2 & T3 & T4 & T5 \\
\hline Aroma & $6,12 \mathrm{ab}$ & $5,86 \mathrm{ab}$ & $5,48 \mathrm{~b}$ & $6,24 \mathrm{a}$ & $6,29 \mathrm{a}$ \\
Sabor & $5,92 \mathrm{a}$ & $5,01 \mathrm{ab}$ & $4,11 \mathrm{~b}$ & $5,80 \mathrm{a}$ & $5,91 \mathrm{a}$ \\
Sabor Picante & $4,45 \mathrm{a}$ & $3,30 \mathrm{bc}$ & $3,12 \mathrm{c}$ & $4,18 \mathrm{a}$ & $3,94 \mathrm{ab}$ \\
Gosto Ácido & $3,31 \mathrm{ab}$ & $3,03 \mathrm{ab}$ & $2,99 \mathrm{ab}$ & $3,39 \mathrm{a}$ & $2,82 \mathrm{~b}$ \\
Sabor Estranho & $1,83 \mathrm{~b}$ & $2,55 \mathrm{ab}$ & $3,65 \mathrm{a}$ & $1,57 \mathrm{~b}$ & $1,24 \mathrm{~b}$ \\
Consistência & $3,36 \mathrm{a}$ & $3,56 \mathrm{a}$ & $3,76 \mathrm{a}$ & $2,93 \mathrm{a}$ & $4,18 \mathrm{a}$ \\
Textura & $3,50 \mathrm{ab}$ & $4,24 \mathrm{a}$ & $4,48 \mathrm{a}$ & $2,84 \mathrm{~b}$ & $3,37 \mathrm{ab}$ \\
Olhaduras mecânicas e/ou gasosas & $2,53 \mathrm{bc}$ & $3,90 \mathrm{ab}$ & $5,55 \mathrm{a}$ & $1,81 \mathrm{c}$ & $2,60 \mathrm{bc}$ \\
Escala Hedônica & $6,35 \mathrm{a}$ & $5,42 \mathrm{ab}$ & $4,42 \mathrm{~b}$ & $6,70 \mathrm{a}$ & $6,35 \mathrm{a}$ \\
\hline
\end{tabular}

T1-Queijos elaborados sem congelamento do leite e da coalhada, (S.C.); T2-Queijos elaborados com congelamento lento da coalhada obtida, (C.L.C.); T3-Queijos elaborados com congelamento rápido da coalhada obtida, utilizando-se nitrogênio líquido, (C.R.C.); T4-Queijos elaborados com congelamento rápido do leite, utilizando-se nitrogênio líquido, (C.R.L.); e T5-Queijos elaborados com congelamento lento do leite, (C.L.L.). Letras diferentes indicam diferenças significativas entre tratamentos pelo teste de Tukey $(\mathrm{p}<0,05)$.

TABELA 2 - Resultados da análise estatística. Estatística calculada e comentários.

\begin{tabular}{lll}
\hline Atributo Sensorial & \multicolumn{1}{c}{ Estatística } & \multicolumn{1}{c}{ Comentário } \\
\hline Aroma & $\mathrm{F}=4,21 ; \mathrm{p}<0,05$ & $(\mathrm{~T} 4=\mathrm{T} 5)>\mathrm{T} 3 ;(\mathrm{T} 1=\mathrm{T} 2)$ intermediários \\
Sabor & $\mathrm{F}=10,29 ; \mathrm{p}<0,05$ & $(\mathrm{~T} 1=\mathrm{T} 4=\mathrm{T} 5)>\mathrm{T} 3 ; \mathrm{T} 2$ intermediário \\
Sabor Picante & $\mathrm{F}=12,68 ; \mathrm{p}<0,05$ & $(\mathrm{~T} 1=\mathrm{T} 4)>(\mathrm{T} 2=\mathrm{T} 3) ; \mathrm{T} 5$ intermediário \\
Gosto Ácido & $\mathrm{F}=3,48 ; \mathrm{p}<0,05$ & Única diferença significativa: (T4 $>\mathrm{T} 5)$ \\
Sabor Estranho & $\mathrm{F}=12,83 ; \mathrm{p}<0,05$ & $(\mathrm{~T} 1=\mathrm{T} 4=\mathrm{T} 5)<\mathrm{T} 3 ; \mathrm{T} 2$ intermediário \\
Consistência & $\mathrm{F}=2,07 ; \mathrm{p}>0,10$ & Tratamentos não diferem \\
Textura & $\mathrm{F}=6,40 ; \mathrm{p}<0,05$ & $(\mathrm{~T} 2=\mathrm{T} 3)>\mathrm{T} 4 ;(\mathrm{T} 1=\mathrm{T} 5)$ intermediários \\
Olhaduras mecânicas e/ou & $\mathrm{F}=11,53 ; \mathrm{p}<0,05$ & $(\mathrm{~T} 1=\mathrm{T} 4=\mathrm{T} 5)<\mathrm{T} 3 ; \mathrm{T} 2$ intermediário \\
gasosas & $\mathrm{F}=9,11 ; \mathrm{p}<0,05$ & $(\mathrm{~T} 1=\mathrm{T} 4=\mathrm{T} 5)>\mathrm{T} 3 ; \mathrm{T} 2$ intermediário \\
Escala Hedônica &
\end{tabular}

T1-Queijos elaborados sem congelamento do leite e da coalhada, (S.C.); T2-Queijos elaborados com congelamento lento da coalhada obtida, (C.L.C.); T3-Queijos elaborados com congelamento rápido da coalhada obtida, utilizando-se nitrogênio líquido, (C.R.C.); T4-Queijos elaborados com congelamento rápido do leite, utilizando-se nitrogênio líquido, (C.R.L.); e T5-Queijos elaborados com congelamento lento do leite (C.L.L.). 
que as médias se situaram entre 3,1 e 4,4 indicando que o produto apresentou sabor picante entre médio e baixo. Com o aumento do tempo de maturação do produto o sabor picante poderia se apresentar mais acentuado.

\section{Gosto ácido}

Praticamente todos os tratamentos apresentaram médias baixas em torno de 3,0, demonstrando que este aspecto desagradável do produto não foi detectado pelos provadores, ou o foi em pequena escala.

\section{Sabor estranho}

Os tratamentos T1, T4 e T5 apresentaram médias muito baixas, sempre menores que 2,0. A maior média ocorreu em T3, ainda assim, com valor médio que pode ser considerado baixo $(3,65)$.

\section{Consistência}

Os tratamentos não diferiram, com médias situando-se entre 3,0 e 4,0 indicando que o produto apresentou consistência entre média e baixa, o que caracteriza consistência macia nos 5 tratamentos.

\section{Textura}

Os tratamentos T2 e T3 apresentaram médias entre 4,0 e 4,5 que corresponderiam a valores medianos na escala, sendo os dois tratamentos com as piores médias. O T4 com média menor que 3,0 apresentou o melhor desempenho, com textura farinácea que pode ser considerada baixa.

\section{Olhaduras mecânicas e ou gasosas}

Os tratamentos com melhor desempenho, T1, T4 e T5, apresentaram médias muito baixas, entre 1,80 e 2,60. O único tratamento com desempenho ruim foi $\mathrm{T} 3 \mathrm{com}$ média 5,55 que representa resultado indesejável para este atributo.

\section{Escala hedônica}

O pior resultado médio representado pelo T3 $(4,42)$ ainda pode ser considerado mediano. Os tratamentos $\mathrm{T} 1$, T4 e T5 tiveram médias boas, sempre superiores a 6,0.

Face aos resultados da análise estatística efetuada para cada variável separadamente, foi possível afirmar que, para o conjunto dos nove atributos sensoriais: T4 teve o melhor desempenho com médias mais altas para aroma, sabor e escala hedônica e médias mais baixas para gosto ácido, sabor estranho e olhaduras mecânicas e ou gasosas. Mostrou, também, média baixa para sabor picante, textura e consistência. Os tratamentos T1 e T5 foram os que mais se aproximaram de T4, dele não diferindo estatisticamente para algumas das variáveis analisadas.

O pior desempenho foi o de T3, ainda assim, com resultados médios que não o desqualificaram totalmente.

\section{CONCLUSÃO}

Os melhores produtos caracterizados por médias mais altas nas provas sensoriais de aroma, sabor e para escala hedônica foram obtidos nos tratamentos T1, T4 e T5.

O pior desempenho foi observado no tratamento T3. Portanto, o queijo estudado poderia utilizar leite de cabra nas condições naturais (T1) nas épocas de pico da produção e utilizar congelamento lento do leite (T5), que seria realizado na época de pico da produção, na entressafra.

\section{AGRADECIMENTOS}

À Fundação de Amparo à Pesquisa do Estado de São Paulo (FAPESP).

À Chr. Hansen pelo fornecimento das culturas láticas.

\section{REFERÊNCIAS BIBLIOGRÁFICAS}

ALBUQUERQUE, L. C. de; CASTRO, M. C. D. Do leite ao queijo de cabra: a história, a tecnologia, o mercado. Juiz de Fora: Concorde, 1996. 162 p.

BONASSI, I. A.; ROÇA, R. O.; GOLDONI, J. S. Avaliação sensorial de queijo Minas Frescal com utilização de leite de cabra congelado e coalhada congelada. In: CONGRESSO BRASILEIRO DE CIÊNCIA E TECNOLOGIA DE ALIMENTOS, 18., 2002, Porto Alegre. Anais... Porto Alegre: UFRGS, 2002.

BRASIL. Ministério da Agricultura, Pecuária e Abastecimento. Secretaria de Defesa Agropecuária. Departamento de Inspeção de Produtos de Origem Animal. Instruçãa normativa DIPOA. Disponível em: <http:// 'www.agricultura.gov.br/dipoà. Acesso em: $20 \mathrm{fev} .2002$.

CORDEIRO, P. R. C. Novas técnicas: verão artificial. Globo Rural, Rio de Janeiro, v. 11, n. 124, p. 7-10, 1996.

CURI, P. R. Metodologia e análise da pesquisa em ciências biológicas. 2. ed. Botucatu: Tipomic, 1998. 263 p.

DUMOULIN, E.; PERETZ, G. Qualité bactériologique du lait cru de chèvre en France. Lait, [S.1.], v. 73, p. 475-483, 1993. 
FURTADO, M. M. Fabricação de queijo de leite de cabra. 6. ed. São Paulo: Nobel, 1985. 126 p.

GAIFAMI, P. I'formaggio di cabra: quad. Contrainformazione Aliment., [S.1.], n. 28, p. 14-18, 1985.

GOMES, M. I. F. V.; BONASSI, I. A.; ROÇA, R. O. Características químicas, microbiológicas e sensoriais de leite de cabra congelado. Ciência e Tecnologia de Alimentos, Campinas, v. 17, p. 111-114, 1997.

INSTITUTE OF FOOD TECHNOLOGISTS. Sensory Evaluation Division. Sensory evaluation guide for testing food and beverage products. Food Technology, Chicago, v. 35 , n. 11, p. 49-58, 1981.

MEILGAARD, M.; CIVILLE, G. V.; CARR, B. T. Sensory evaluation techniques. Boca Raton: CRC, 1990. 281 p.

RAMOS, M.; JUAREZ, M. Current research on goats' milk in Spain. Lait, [S.1.], v. 73, p. 417-424, 1993.

RIBEIRO, S. D. A. Produção intensiva de caprinos. Revista Brasileira de Reprodução Animal, São Paulo, v. 1, p. 143149, 1993.

ROÇA, R. O.; BONASSI, I. A. Seleção de provadores para produtos cárneos. In: CONGRESSO BRASILEIRO DE
CIÊNCIA E TECNOLOGIA DE ALIMENTOS, 7., 1985, Itabuna/Ilhéus. Anais... Itabuna: Sociedade Brasileira de Ciência e Tecnologia de Alimentos, 1985. p. 83.

SÃO PAULO. Secretaria da Agricultura e Abastecimento. Resolução ${ }^{\circ}{ }^{93}$, de 14 de outubro de 1993. Dispõe sobre a produção do leite de cabra e seus derivados em condições artesanais. Diário Oficial do Estado de São Paulo, São Paulo, v. 103, n. 193, p. 11-12, 1993. Seção 1.

SINGH, S.; RAO, K. H.; KANAWJIA, S. K.; SABIKHI, L. Goat milk products technology: a review. Indian Journal of Dairy Science, [S.1.], v. 45, n. 11, p. 572-587, 1992.

YAMASAKI, H. R.; TANIGUCH, T.; MASUDA, T.; MORICHI, T. Extension in the supply period of Sainte Maure cheese by use frozen curd. Journal of the Japanese Societ for Food Science and Technology, Tokio, v. 48, n. 6, p. 444-448, 2001

ZAPICO, P.; GAYA, P.; NUÑEZ, M.; MEDINA, M. Activity of goat's milk lactoperoxidase system on Pseudomonas fluorescens and Escherichia coli at refrigeration temperatures. Journal Food Protection, Des Moines, v. 58, p. 1136-1138, 1995. 